\title{
Giant chronic expanding hematoma in the chest identified 25 years after a blunt chest trauma
}

\author{
WEI DAI ${ }^{1}$, XIANG ZHUANG ${ }^{1}$, QIANG LI ${ }^{1}$, PING XIAO ${ }^{1}$, YI SHEN ${ }^{1}$ and PING ZHENG $^{2}$ \\ Departments of ${ }^{1}$ Thoracic Surgery and ${ }^{2}$ Pathology, Sichuan Cancer Hospital, Chengdu, Sichuan 610041, P.R. China
}

Received July 27, 2015; Accepted February 3, 2016

DOI: $10.3892 / \mathrm{mco} .2016 .774$

\begin{abstract}
We herein report the case of a 42-year-old man who presented with a huge intrathoracic mass that had grown over a period of 25 years. The initial symptom caused by the mass was dull pain in the chest. T2-weighted magnetic resonance imaging revealed a mosaic pattern of various signal intensities, indicating a chronic expanding hematoma. The mass was completely resected surgically. For patients who present with a slowly growing mass, particularly those with a history of tuberculous pleuritis, chest surgery or trauma, a chronic expanding hematoma should be taken into consideration. Surgical resection is the first choice of treatment for a chronic expanding hematoma caused by a blunt chest trauma.
\end{abstract}

\section{Introduction}

A chronic expanding hematoma of the chest is a rare condition that often occurs months to years after tuberculous pleuritis or thoracic surgery, and rarely after a blunt chest trauma (1-3). Chest injury due to blunt chest trauma may result in a hemorrhage or an acute intrathoracic hematoma, which is often naturally reabsorbed and rarely causes serious problems. Under rare conditions, however, the mass slowly grows and enlarges inside the chest. Surgical removal is the main treatment for thoracic hematomas. However, complete resection is not easily achieved, mainly due to the presence of dense fibrous adhesions to adjacent tissues. We herein report the case of a patient with a huge chronic expanding hematoma in the chest, which had grown over a 25 -year period following a blunt chest trauma. The patient was successfully treated by surgical resection.

\section{Case report}

A 42-year-old man with a dull pain in the chest was admitted to the Sichuan Cancer Hospital (Chengdu, China). Apart from

Correspondence to: Dr Xiang Zhuang, Department of Thoracic Surgery, Sichuan Cancer Hospital, 55 South Renmin Road, 4th section, Chengdu, Sichuan 610041, P.R. China

E-mail: uniondavii@sina.com

Key words: chronic expanding hematoma, chest injury, diagnosis, surgery chest pain, no other symptoms or signs were found. The patient denied any history of thoracic surgery or pulmonary diseases, including tuberculosis, but recalled an incident of blunt chest trauma. The patient was injured in the chest during a fight 25 years ago, but he did not seek medical help at that time. Chest $\mathrm{X}$-ray revealed a huge mass shadow in the anterior mediastinum. Chest computed tomography (CT) revealed an intrathoracic mass sized $10.2 \times 13.3 \times 17.9 \mathrm{~cm}$, without calcification, which tightly adhered to the left pericardium. A left moderate pleural effusion was also detected on CT imaging (Fig. 1A and B). T2-weighted magnetic resonance imaging (MRI) revealed a variety of signal intensities in the mass, appearing as a mosaic pattern (Fig. 1C). Diagnostic thoracocentesis was performed to determine the characteristics of the left pleural effusion, and only erythrocytes and a few inflammatory cells were detected by cytological examination. Subsequently, a CT-guided needle biopsy was performed, but the results were negative.

The mass was then considered to be a benign or low-to-moderate malignant tumor, such as a teratoma, or chronic empyema. Most of the lesion was located in the left thorax and complete resection of the lesion appeared to be feasible, based on the preoperative imaging evaluation. Therefore, a left posterolateral thoracotomy through the 5 th intercostal space was performed. There were no pleural adhesions. The mass was located in the left anterior mediastinum and had a thickened, hard capsule, which was loosely attached to the diaphragm, but was tightly adherent to the thymus, pericardium and upper lobe of the left lung. The mass was completely removed, with most of the thymus and left parietal pericardium, and part of the upper lobe of the left lung (Fig. 2A). The operative time was $140 \mathrm{~min}$ and the total intraoperative blood loss was $250 \mathrm{ml}$.

On macroscopic examination, the resected mass was surrounded by a dense fibrous capsule, filled with hemorrhagic material and necrotic tissue (Fig. 2A-C), which was further confirmed by histopathological analysis (Fig. 2D). There was no evidence of malignancy or infection within the mass. Finally, the patient was diagnosed with a chronic expanding hematoma. The postoperative course was uneventful. There was no sign of recurrence during the 6 months of follow-up.

\section{Discussion}

A chronic expanding hematoma was first described by Reid et al as a rare clinicopathological entity, characterized 

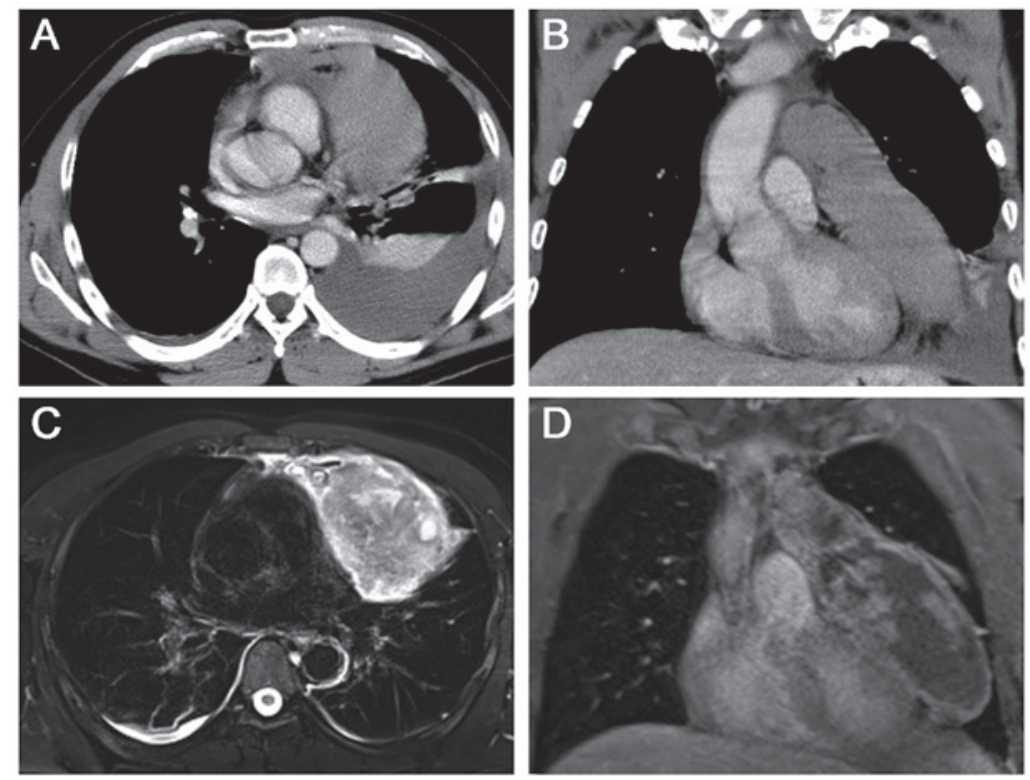

Figure 1. (A and B) Chest computed tomography scan revealed a huge intrathoracic mass and moderate pleural effusion in the left pleural cavity. (C) T2-weighted magnetic resonance imaging demonstrated a huge well-defined mass with a mixture of high- and low-signal intensity areas in the chest. (D) T1-weighted images showed a mass with a mildly high signal intensity in the left anterior pericardium.
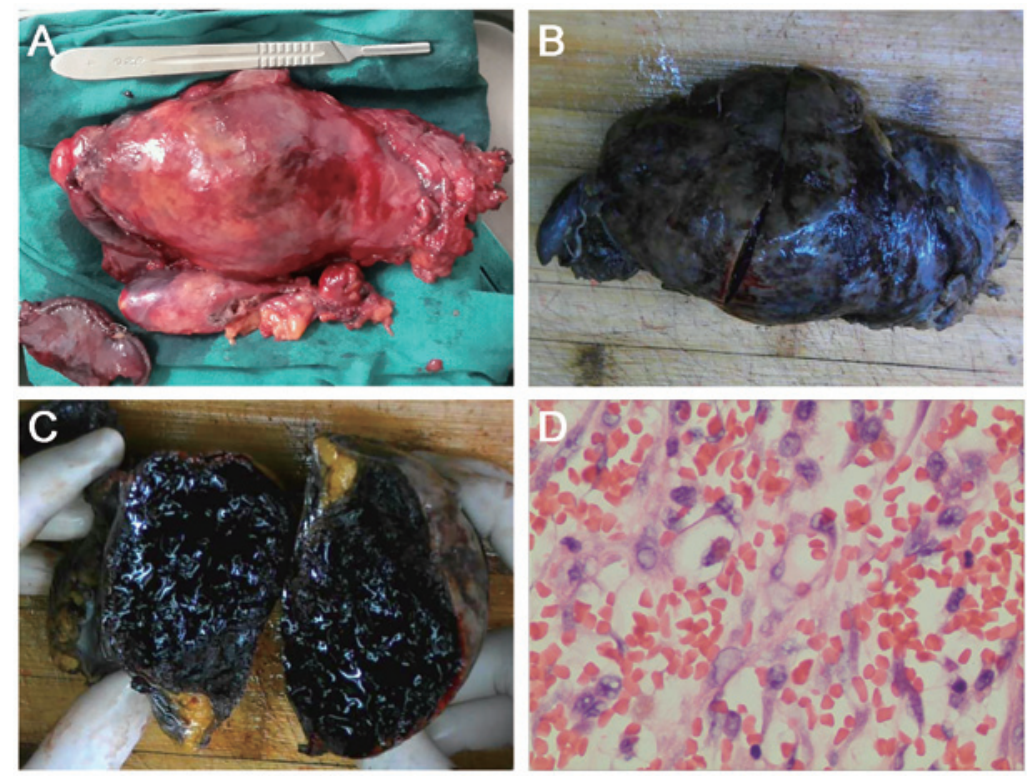

Figure 2. (A-C) On macroscopic examination, the resected mass had a dense fibrous capsule, which was filled with hemorrhagic material and necrotic tissue. (D) Histological analysis revealed an old hematoma surrounded by fibrous tissue, with dilated microvessels and blood stasis within the hematoma.

by its persistence and increasing size after the initial hemorrhage (4). Chronic expanding hematoma may occur in various locations. Although huge chronic expanding hematomas in the chest have been previously reported, they were usually caused by tuberculous pleuritis or thoracic surgery (1-3), whereas blunt chest trauma as a causative factor is rare. It remains unclear why chronic expanding hematomas grow continuously. Labadie et al proposed a theory that chronic expanding hematoma formation is a complex process of initial hemorrhage followed by repeated organization and hemorrhage from new microvessels beneath the capsule (5). In the present case, the patient suffered an initial hemorrhage in his chest caused by a blunt chest trauma 25 years ago. The small hematoma did not resolve naturally, and it grew slowly, with repeated organization and rehemorrhage. Various factors, such as heart beating, respiratory movements and coughing under negative pleural pressure, likely promoted the growth of the hematoma, finally resulting in the formation of a huge mass.

Chronic expanding hematoma in the chest is difficult to diagnose due to its rarity and slow growth, particularly for patients without any history of surgery, tuberculosis or trauma (6). Although a definitive diagnosis of a chronic expanding hematoma mainly depends on histopathology, MRI plays an important role in the differential diagnosis from other common mediastinal tumors, such as thymoma, teratoma and lymphoma. In the present case, the hematoma displayed 
various signal intensities on T2-weighted images and exhibited a mosaic pattern, which was previously reported as a characteristic feature of chronic expanding hematomas $(6,7)$. The various intensities on the MRI images corresponded to fresh and old blood, which was the result of repeated hemorrhages over time. Based on this case report and the currently available literature, the characteristics of chronic expanding hematomas in the chest are summarized as follows: i) The patient has a mass over a relatively long time period; ii) a history of tuberculous pleuritis, chest surgery or trauma; iii) a mosaic pattern of various signal intensities on T2-weighted MRI images is observed; and iv) no positive results are found by preoperative biopsy examination. Thus, a chronic expanding hematoma of the chest should be taken into consideration if the case is consistent with the abovementioned characteristics.

Surgical resection is the main treatment for patients with a chronic expanding hematoma to relieve the pressure on adjacent organs. However, as reported previously, surgical resection of these hematomas may be challenging, including a long operative time, failure of complete resection due to the strong adhesion of the hematoma to the surrounding tissues, and massive intraoperative blood loss. Patients have been reported to experience a blood loss of $>1,000 \mathrm{ml}$ in the majority of the cases $(1-3,6)$, and massive bleeding of $>10,000 \mathrm{ml}$ in occasional cases (3). In the present case, the intrathoracic lesion was completely removed with a total operative time of $140 \mathrm{~min}$ and a total blood loss of only $250 \mathrm{ml}$, possibly because the patient had no history of a thoracic surgery or tuberculosis, which often cause severe pleural adhesions and abundant neovascular proliferation. Therefore, surgical resection is the first choice of treatment for a chronic expanding hematoma caused by a blunt chest trauma.

For patients who present with slowly growing intrathoracic masses, particularly those with a history of chest trauma and a mosaic pattern of various signal intensities on T2-weighted MRI images, the diagnosis of a chronic expanding hematoma should be taken into consideration and surgical resection is the first choice of treatment.

\section{References}

1. Hanagiri T, Muranaka H, Hashimoto M, Nishio T, Sakai S, Ono M, Toyoshima S and Nagashima A: Chronic expanding hematoma in the chest. Ann Thorac Surg 64: 559-561, 1997.

2. Muramatsu T, Shimamura M, Furuichi M, Ishimoto S, Ohmori K and Shiono M: Treatment strategies for chronic expanding hematomas of the thorax. Surg Today 41: 1207-1210, 2011.

3. Ogata J, Minami K, Nakamura M, Horishita T and Sata T: The management of extirpation of chronic expanding hematoma after thoracoplasty in the chest. Masui 53: 1286-1289, 2004 (In Japanese).

4. Reid JD, Kommareddi S, Lankerani M and Park MC: Chronic expanding hematomas. A clinicopathologic entity. JAMA 244: 2441-2442, 1980.

5. Labadie EL and Glover D: Physiopathogenesis of subdural hematomas. Part 1: Histological and biochemical comparisons of subcutaneous hematoma in rats with subdural hematoma in man. J Neurosurg 45: 382-392, 1976.

6. Kuronuma K, Ootake S, Ikeda K, Taniguchi M, Takezawa C and Takahashi H: Chronic expanding hematoma in the chest. Intern Med 47: 1411-1414, 2008.

7. Akata S, Ohkubo Y, Jinho P, Saito K, Yamagishi T, Yoshimura M, Kotake F, Kakizaki D and Abe K: MR features of a case of chronic expanding hematoma. Clin Imaging 24: 44-46, 2000. 